\title{
MIXED CONVECTION OF A CU-AG/WATER HYBRID NANOFLUID ALONG A VERTICAL POROUS CYLINDER VIA MODIFIED TIWARI-DAS MODEL
}

\author{
Saeed Dinarvand*, Mohammadreza Nademi Rostami \\ Department of Mechanical Engineering, Islamic Azad University, Central \\ Tehran Branch, Tehran, Iran
}

[Received: July 14, 2018. Accepted: May 13, 2019]

doi: 10.7546/JTAM.49.19.02.05

\begin{abstract}
Herein, a Cu-Ag/water hybrid nanofluid is analytically modeled to develop the problem of the MHD axisymmetric mixed convective stagnationpoint flow along a vertical porous cylinder. A new mathematical model has been presented and formulated based on Tiwari-Das nanofluid scheme. Using similarity transformations, the governing PDEs are altered into dimensionless ODEs, which is solved numerically using bvp4c function from MATLAB. The thermal characteristics of hybrid nanofluid are found to be higher relative to mono-nanofluid and base fluid, respectively. Besides, the results indicate that dual solutions exist for hybrid nanofluid flow in both assisting and opposing regimes. We conclude that the developed model can be used with great confidence to study the flow and heat transfer of hybrid nanofluid in various problems.
\end{abstract}

KEY WORDS: analytic model, dual solutions, hybrid nanofluid, mixed convection, MHD

\section{NOMENCLATURE}

$\begin{array}{llll}a & \text { radius of cylinder } & U_{\infty} & \text { characteristic velocity } \\ B_{0} & \text { uniform magnetic field } & u, w & \text { velocity components along } x, r \\ C_{f} & \text { skin friction coefficient } & V_{w} & \text { permeability parameter } \\ C_{P} & \text { specific heat at constant pressure } & V_{w}^{*} & \text { uniform surface mass flux } \\ g & \text { gravity acceleration } & x, r & \text { axisymmetric cylindrical coordinates } \\ G r & \text { local Grashof number } & \bar{x} & \text { rescale axial coordinate } \\ k & \text { thermal conductivity } & \Delta T & \text { characteristic temperature } \\ \ell & \text { characteristic length } & \sigma & \text { electrical conductivity } \\ M & \text { magnetic parameter } & \phi & \text { nanoparticle volume fraction } \\ N u & \text { local Nusselt number } & \gamma & \text { curvature parameter } \\ P & \text { hybrid nanofluid pressure } & \eta & \text { similarity variable } \\ P r & \text { Prandtl number } & f(\eta) & \text { dimensionless stream function } \\ q w & \text { surface heat flux } & \theta(\eta) & \text { dimensionless temperature distribution } \\ R e & \text { local Reynolds number } & \lambda & \text { mixed convection parameter } \\ T & \text { temperature } & \mu & \text { dynamic viscosity } \\ U(x) & \text { mainstream velocity } & v & \text { kinematic viscosity }\end{array}$

\footnotetext{
${ }^{*}$ Corresponding author e-mail: sae.dinarvand@iauctb.ac.ir ; saeed_dinarvand@yahoo.com
} 


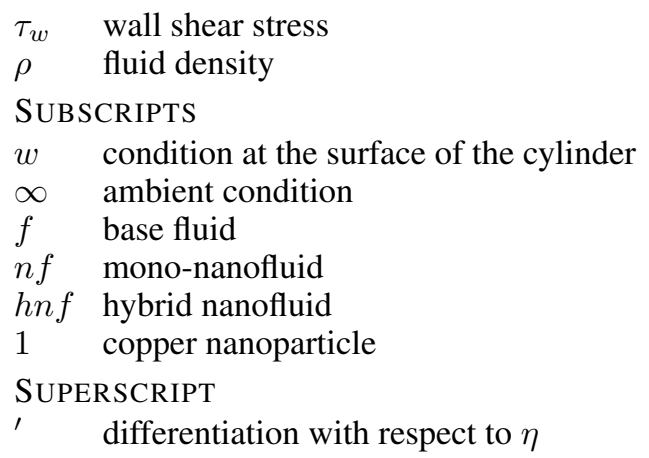

\section{INTRODUCTION}

Nanofluids are engineered by suspending nanoparticles with average sizes below $100 \mathrm{~nm}$ in traditional heat transfer fluids such as water, oil, ethylene glycol, etc. [1-3]. The goal of nanofluids is to achieve the highest possible thermal properties at the smallest possible concentrations (preferably $<1 \%$ by volume) by uniform dispersion and stable suspension of nanoparticles (preferably $<10 \mathrm{~nm}$ ) in host fluids. To achieve this goal it is vital to understand how nanoparticles enhance energy transport in liquids [4]. Kasaeian et al. [5] have presented a comprehensive study on the simultaneous application of nanofluids and porous media for heat transfer enhancement purposes in thermal systems with different structures, flow regimes, and boundary conditions. It was found that Tiwari and Das [6] and Buongiorno [7] models were the most popular models used to simulate the nanofluid flow in porous media. For example, the first model have been used by Ismael et al. [8] and Chamkha and Ismael [9] to numerically analyze the entropy generation due to conjugate natural convectionconduction heat transfer in a square domain consist of porous cavity heated by a triangular solid wall saturated with a $\mathrm{CuO}$-water nanofluid and natural convection heat transfer in a differentially heated and vertically partially layered porous cavity filled with a $\mathrm{Cu}$-water nanofluid, respectively. On the other hand, the second model was applied to a boundary layer problem for the natural convection past a nonisothermal vertical plate in a porous medium filled with a nanofluid by Gorla and Chamkha [10]. By the way, Zaraki et al. [11] were also theoretically exploited Buongiorno's model to develop the natural convection heat and mass transfer of nanofluids over a flat plate considering size, shape, different types of nanoparticles, base fluid and working temperature. Hybrid nanofluids are very new kind of nanofluids, which can be prepared by suspending (i) different types (two or more than two) of nanoparticles in base fluid, and (ii) hybrid (composite) nanoparticles in base fluid [12]. In 2017, Mehryan et al. [13] have studied the free convective heat transfer of the $\mathrm{Al}_{2} \mathrm{O}_{3}-\mathrm{Cu}$ water hybrid nanofluid in a square cavity filled with a porous medium. The actual 
available experimental data for the thermal conductivity and the dynamic viscosity of hybrid nanofluids were directly utilized in this study. In most cases, the average Nusselt number was decreasing function of the volume fraction of nanoparticles. However, at the same year, Chamkha et al. [14] worked on the unsteady conjugate natural convection in a semicircular cavity with a solid shell of finite thickness filled with the same hybrid nanofluid and the obtained results have revealed essential heat transfer enhancement at solid-fluid interface with addition of nanoparticles. Moreover, in 2018, Ghalambaz et al. [15] have focused on the conjugate free convection inside a porous square cavity occupied with $\mathrm{Ag}-\mathrm{MgO}$ hybrid nanofluid using the local thermal non-equilibrium model. Further, the natural convection of multi-wall carbon nanotubes-Iron oxide nanoparticles/water hybrid nanofluid inside an inverse T shaped enclosure have been investigated by Izadi et al. [16] using Lattice Boltzmann Method.

Mixed convection is a coupled phenomenon of free and forced convection. As general case, the mixed convection is formed simultaneously by an outer forcing flow and inner volumetric (mass) force, while the latter is caused by the no-uniform density distribution of a fluid medium in a gravity field. Generally, for the assisting flow, free and forced convections are in the same direction, and the mixed convection parameter is positive. But, the opposite behavior is valid for opposing flow [17]. Some examples include solar receivers exposed to wind currents, electronic devices cooled by fans, various lid-driven cavities, nuclear reactors cooled during emergency shutdown, flows in the ocean and in the atmosphere, etc. [18-23]. RamReddy et al. [24] intended to illustrate the influence of the prominent Soret effect on mixed convection heat and mass transfer in the boundary layer region of a semi-infinite vertical flat plate in a nanofluid under the convective boundary conditions with help of Buongiorno's model. Dinarvand et al. [25] investigated the steady axisymmetric mixed convective stagnation-point flow of an incompressible electrically conducting nanofluid over a vertical circular cylinder imposed by a transverse magnetic field based on Tiwari-Das nanofluid model.

Ramachandran et al. [19] studied the steady laminar mixed convection in twodimensional stagnation point flows around vertical surfaces by considering both cases of an arbitrary wall temperature and arbitrary surface heat flux variations. They found that a reverse flow develops in the buoyancy opposing flow region, and dual solutions are found to exist for a certain range of the buoyancy parameter. The existence of dual solutions for both assisting and opposing flows was reported by Ishak et al. [20] and Ridha [26]. They explored the mixed convection boundary layer flow with a constant magnetic field normal to the vertical plate.

The aim of the present article is to study the steady MHD axisymmetric mixed convective stagnation-point flow of an aqueous hybrid nanofluid (considering copper 
and silver as nanoparticles) over a vertical permeable cylinder through Tiwari-Das nanofluid model. Using appropriate similarity transformation [27], non-linear governing partial differential equations are altered into dimensionless non-linear ODEs, which are then solved numerically by a finite difference scheme from MATLAB that implements the 3-stage Lobatto IIIa formula. Finally, our computational results present through graphs and Tables.

\section{Model Development and Mathematical Formulation}

Consider the steady, axisymmetric mixed convective stagnation-point flow of an incompressible electrically conducting hybrid nanofluid over a vertical permeable cylinder in the presence of transverse magnetic field as shown in Fig. 1.

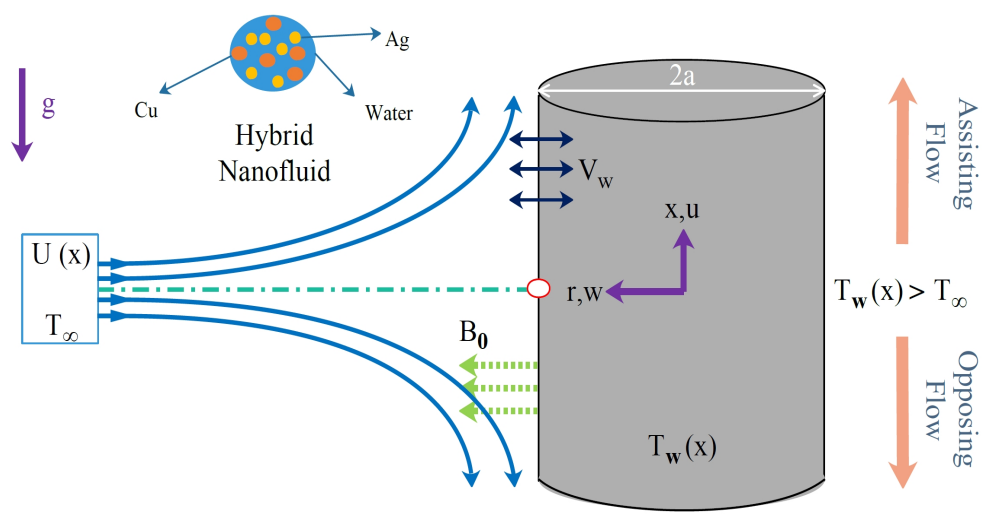

Fig. 1. (Color online) The schematic diagram of problem and coordinate system.

We have considered copper $(\mathrm{Cu})$ and silver $(\mathrm{Ag})$ as nanoparticles with water as base fluid. In our model, copper is initially scattered into the base fluid to make nanofluid $\mathrm{Cu} /$ water. Besides, to develop the targeted hybrid nanofluid $\mathrm{Cu}-\mathrm{Ag} /$ water, silver is dispersed in $\mathrm{Cu} /$ water nanofluid. Table 1 shows applied models for thermophysical properties of the nanofluid and hybrid nanofluid [28-31]. Moreover, in Table 2 we can see thermophysical properties of the base fluid and the nanoparticles [31-36]. Moreover, in present study, it is assumed that nanoparticles and base fluid are in thermal equilibrium and no slip occurs between them.

By using the Tiwari-Das mathematical model [6], the governing boundary layer equations of the problem can be written as $[25,36]$

$$
\frac{\partial}{\partial x}(r u)+\frac{\partial}{\partial r}(r w)=0,
$$



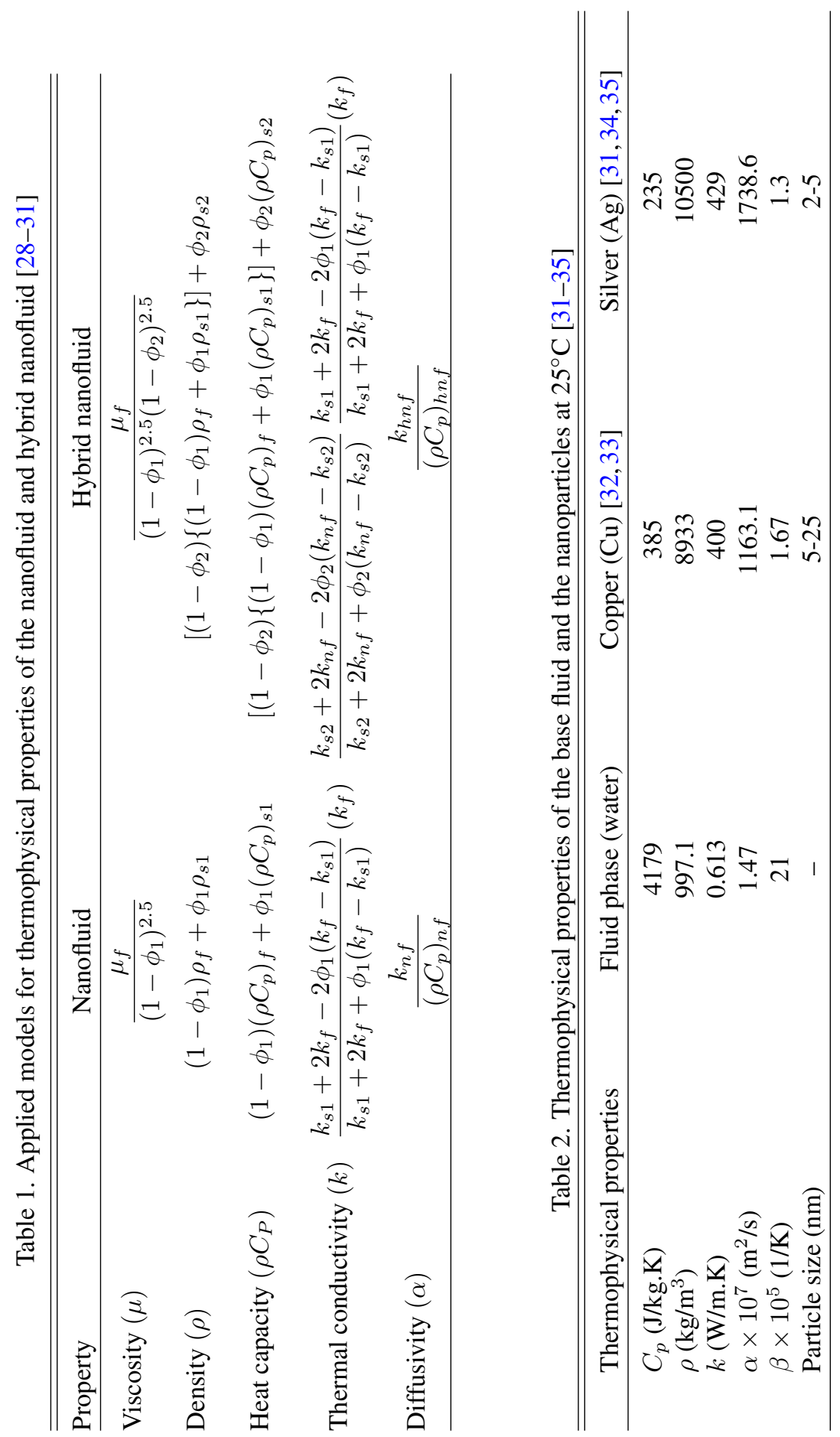


$$
\begin{aligned}
& u \frac{\partial u}{\partial x}+w \frac{\partial u}{\partial r}=U \frac{\partial U}{\partial x}+\frac{\mu_{h n f}}{\rho_{h n f}}\left(\frac{\partial^{2} u}{\partial r^{2}}+\frac{1}{r} \frac{\partial u}{\partial r}\right)+\frac{\sigma B_{0}^{2}}{\rho_{h n f}}(U-u) \\
& +\frac{\left[\left(1-\phi_{2}\right)\left\{\left(1-\phi_{1}\right) \rho_{f} \beta_{f}+\phi_{1} \rho_{s 1} \beta_{s 1}\right\}\right]+\phi_{2} \rho_{s 2} \beta_{s 2}}{\rho_{h n f}} g\left(T-T_{\infty}\right), \\
& u \frac{\partial T}{\partial x}+w \frac{\partial T}{\partial r}=\alpha_{h n f}\left(\frac{\partial^{2} T}{\partial r^{2}}+\frac{1}{r} \frac{\partial T}{\partial r}\right),
\end{aligned}
$$

subject to the boundary conditions

$$
u=0, \quad w=V_{w}^{*}, \quad T=T_{w}(x)=T_{\infty}+\Delta T(x / \ell) \quad \text { at } r,=a
$$

(4)

$$
u=U(x) \rightarrow U_{\infty}(x / \ell), \quad T \rightarrow T_{\infty} \quad \text { as } r \rightarrow \infty .
$$

We notice that, $V_{w}^{*}<0$ corresponds to suction and $V_{w}^{*}>0$ corresponds to injection. Let us to consider the following similarity transformations [25]:

$$
\psi=\left(\frac{U_{\infty} \nu_{f} a^{2}}{\ell}\right)^{1 / 2} x f(\eta), T-T \infty=\Delta T\left(\frac{x}{\ell}\right) \theta(\eta), \eta=\frac{r^{2}-a^{2}}{2 v_{f} \ell}\left(\frac{U_{\infty} \nu_{f} \ell}{a^{2}}\right)^{1 / 2},
$$

where $\psi$ is the stream function in which $u=(1 / r)(\partial \psi / \partial r)$ and $w=-(1 / r)(\partial \psi / \partial x)$. Substituting Eq. (5) into Eqs. (2) and (3), we obtain a system of dimensionless nonlinear ordinary differential equations

$$
\begin{aligned}
& \text { (6) } C_{1}\left[(1+2 \gamma \eta) f^{\prime \prime \prime}+2 \gamma f^{\prime \prime}\right]+f f^{\prime \prime}-f^{\prime 2}+1+C_{2} M\left(1-f^{\prime}\right)+C_{3} \lambda \theta=0, \\
& C_{4}\left[(1+2 \gamma \eta) \theta^{\prime \prime}+2 \gamma \theta^{\prime}\right]+\operatorname{Pr}\left(f \theta^{\prime}-f^{\prime} \theta\right)=0, \\
& C_{1}=\frac{1}{\left(1-\phi_{1}\right)^{2.5}\left(1-\phi_{2}\right)^{2.5}\left(\left[\left(1-\phi_{2}\right)\left\{\left(1-\phi_{1}\right)+\phi_{1} \frac{\rho_{s 1}}{\rho_{f}}\right\}\right]+\phi_{2} \frac{\rho_{s 2}}{\rho_{f}}\right)}, \\
& C_{2}=\frac{1}{\left[\left(1-\phi_{2}\right)\left\{\left(1-\phi_{1}\right)+\phi_{1} \frac{\rho_{s 1}}{\rho_{f}}\right\}\right]+\phi_{2} \frac{\rho_{s 2}}{\rho_{f}}}, \\
& C_{3}=\frac{\left[\left(1-\phi_{2}\right)\left\{\left(1-\phi_{1}\right)+\phi_{1} \frac{\rho_{s 1}}{\rho_{f}} \frac{\beta_{s 1}}{\beta_{f}}\right\}\right]+\phi_{2} \frac{\rho_{s 2}}{\rho_{f}} \frac{\beta_{s 2}}{\beta_{f}}}{\left[\left(1-\phi_{2}\right)\left\{\left(1-\phi_{1}\right)+\phi_{1} \frac{\rho_{s 1}}{\rho_{f}}\right\}\right]+\phi_{2} \frac{\rho_{s 2}}{\rho_{f}}}, \\
& C_{4}=\frac{k_{h n f}}{k_{f}} \\
& {\left[\left(1-\phi_{2}\right)\left\{\left(1-\phi_{1}\right)+\phi_{1} \frac{\left(\rho C_{p}\right)_{s 1}}{\left(\rho C_{p}\right)_{f}}\right\}\right]+\phi_{2} \frac{\left(\rho C_{p}\right)_{s 2}}{\left(\rho C_{p}\right)_{f}}}
\end{aligned}
$$


subject to the boundary conditions

$$
\begin{gathered}
f(0)=V_{w}, \quad f^{\prime}(0)=0, \quad \theta(0)=1, \\
f^{\prime}(\infty) \rightarrow 1, \quad \theta(\infty) \rightarrow 0 .
\end{gathered}
$$

where $\operatorname{Pr}=\nu_{f} / \alpha_{f}$ is the Prandtl number, and other governing parameters are

(10) $\lambda=\frac{G r}{R e^{2}}, \quad \gamma=\left(\frac{\nu_{f} \ell}{U_{\infty} a^{2}}\right)^{1 / 2}, \quad V_{w}=-\left(\frac{\ell}{\nu_{f} U_{\infty}}\right)^{1 / 2} V_{w}^{*}, \quad M=\frac{\sigma B_{0}^{2}}{\rho_{f}\left(U_{\infty} / \ell\right)}$,

in which $G r=g \beta_{f} \Delta T \ell^{3} / \nu_{f}^{2}$ is the local Grashof number and $R e=U_{\infty} \ell / \nu_{f}$ is the local Reynolds number. It should be mentioned that $\lambda>0$ corresponds to assisting flow, $\lambda<0$ corresponds to opposing flow and $\lambda=0$ corresponds to forced convection flow. Moreover, $V_{w}<0$ is for mass injection and $V_{w}>0$ is for mass suction at the cylinder surface.

The skin friction coefficient $C_{f}$ and the local Nusselt number $N u$ are defined as [25]

$$
C_{f}=\frac{\tau_{w}}{\rho_{f} U_{\infty}^{2}}, \quad N u=\frac{\ell q_{w}}{k_{f} \Delta T} .
$$

Here, $\tau_{w}$ is the surface shear stress and $q_{w}$ is the surface heat flux which are introduced by

$$
\tau_{w}=\mu_{h n f}\left(\frac{\partial u}{\partial r}\right)_{r=a}, \quad q_{w}=-k_{h n f}\left(\frac{\partial T}{\partial r}\right)_{r=a} .
$$

By Eqs. (5), (11) and (12), we have

$$
R e^{1 / 2} C_{f}=\frac{\bar{x}}{\left(1-\varphi_{1}\right)^{2.5}\left(1-\varphi_{2}\right)^{2.5}} f^{\prime \prime}(0), \quad R e^{-1 / 2} N u=-\frac{k_{h n f}}{k_{f}} \bar{x} \theta^{\prime}(0)
$$

where $\bar{x}=x / \ell$.

\section{Numerical SOLUtion AND VAlidation}

The governing Eqs. (6) and (7) subject to the boundary conditions (8) and (9) are solved numerically for some values of the governing parameters $\phi_{1}, \phi_{2}, \lambda, \gamma, M, V_{w}$ and $\operatorname{Pr}$ using the function bvp4c from MATLAB software (see Shampine et al. [37]). The function bvp4c is a finite difference code that implements the 3-stage Lobatto IIIa formula. This is a collocation formula and the collocation polynomial gives us a $C^{1}$ - continuous solution, which is fourth order accurate uniformly in the interval where the function is integrated. In this approach, we have chosen a suitable finite 
value of $\eta \rightarrow \infty$ namely $\eta=\eta_{\infty}$ between 3 and 10, and the relative tolerance was set as default $\left(10^{-3}\right)$.

To validate our numerical procedure, Table 3 shows the value of the skin friction coefficient and the local heat transfer rate for a special case (copper/water nanofluid with different values of $\phi_{1}$ and $\lambda$ when $\operatorname{Pr}=6.2, \gamma=1, M=V_{w}=\phi_{2}=0$ based on the first solution) that is obtained and compared with the results of Dinarvand et al. [25]. Moreover, Table 4 shows the comparison of the values of the skin friction coefficient and the local heat transfer rate based on the second solution for another special case (i.e. pure water with different values of $\operatorname{Pr}$ and $\lambda$, when $\gamma=M=V_{w}=$ $\phi_{1}=\phi_{2}=0$ based on the second solution). It is seen that the present results are in good agreement with solutions obtained by Ishak et al. [20], Grosan et al. [38] and Dinarvand et al. [25]. Thus, it can be concluded that the developed method can be used with great confidence to study the heat and fluid flow of the present problem.

Table 3. The influence of $\phi_{1}$ and $\lambda$ on the skin friction coefficient and the local heat transfer rate based on the first solution for copper-water nanofluid, when $\operatorname{Pr}=6.2, \gamma=1, M=$ $V_{w}=0$ and $\phi_{2}=0$.

\begin{tabular}{ccccccc}
\hline \hline & & \multicolumn{2}{c}{$(1 / \bar{x}) R e^{1 / 2} C_{f}$} & & \multicolumn{2}{c}{$(1 / \bar{x}) R e^{-1 / 2} N u$} \\
\cline { 3 - 4 } \cline { 6 - 6 }$\lambda$ & $\phi_{1}$ & Ref. [25] & Present study & & Ref. [25] & Present study \\
\hline \multirow{2}{*}{0} & 0.0 & 1.70762 & 1.71067 & & 2.15173 & 2.15300 \\
& 0.1 & 2.51214 & 2.51318 & & 2.69666 & 2.69711 \\
& 0.2 & 3.46828 & 3.46936 & & 3.26939 & 3.26987 \\
& & & & & \\
5 & 0.0 & 3.02004 & 3.02257 & & 2.40936 & 2.41023 \\
& 0.1 & 3.83608 & 3.83694 & & 2.92285 & 2.92326 \\
& 0.2 & 4.84840 & 4.84887 & & 3.47668 & 3.47738 \\
\hline
\end{tabular}

Table 4. The skin friction coefficient and the local heat transfer rate based on the second solutions for various values of $\operatorname{Pr}$ and $\lambda$, when $\gamma=0$ (flat plate), $M=0, V_{w}=0$ and $\phi_{1}=\phi_{2}=0$.

\begin{tabular}{lcccccc}
\hline \hline Quantities & $P r$ & $\lambda$ & Ref. [20] & Ref. [38] & Ref. [25] & Present study \\
\hline$(1 / \bar{x}) R e^{1 / 2} C_{f}$ & 6.2 & -2 & - & -0.5956 & -0.595628 & -0.616678 \\
& 6.2 & -1 & - & -0.3287 & -0.328736 & -0.373731 \\
& 6.2 & 1 & 0.6512 & - & 0.651291 & 0.611950 \\
$(1 / \bar{x}) R e^{-1 / 2} N u$ & 6.2 & -2 & - & - & -1.001238 & -1.001389 \\
& 6.2 & -1 & - & -1.8957 & -1.895751 & -1.895728 \\
& 6.2 & 1 & 2.0516 & - & 2.051632 & 2.135455 \\
\hline
\end{tabular}




\section{RESUlTS AND DisCUSSION}

Herein, the effect of aforementioned governing parameters on the flow and heat transfer characteristics, have been displayed in graphical forms. At first, it will be useful to determine ranges of governing parameters in the present study as shown in Table 5.

Table 5. The applied range of governing parameters in the present analysis.

\begin{tabular}{lcc}
\hline \hline Governing parameter & Symbol & Range \\
\hline Volume fraction of first nanoparticle & $\phi_{1}$ & $0 \leq \phi_{1} \leq 0.2$ \\
Volume fraction of second nanoparticle & $\phi_{2}$ & $0 \leq \phi_{2} \leq 0.1$ \\
Mixed convection parameter & $\lambda$ & $-20 \leq \lambda \leq 20^{*}$ \\
Curvature parameter & $\gamma$ & $0 \leq \gamma \leq 5$ \\
Magnetic parameter & $M$ & $0 \leq M \leq 4$ \\
Permeability parameter & $V_{w}$ & $-0.8 \leq V_{w} \leq 0.8$ \\
Prandtl number & $P r$ & $0.7 \leq P r \leq 6.2$ \\
\hline
\end{tabular}

${ }^{*}$ Except for some items related to critical values of $\lambda$ (see section 4.1).

\subsection{MultiPLE SOLUTIONS OF HYBRID NANOFLUID FLOW}

Figures 2 and 3 respectively show the variation of $f^{\prime \prime}(0)$ and $-\theta^{\prime}(0)$ for pure water, copper-water nanofluid and copper-silver/water hybrid nanofluid versus $\lambda$ for $M=1$, $V_{w}=0.5, \gamma=2, \operatorname{Pr}=6.2$ and the determined values of nanoparticles volume

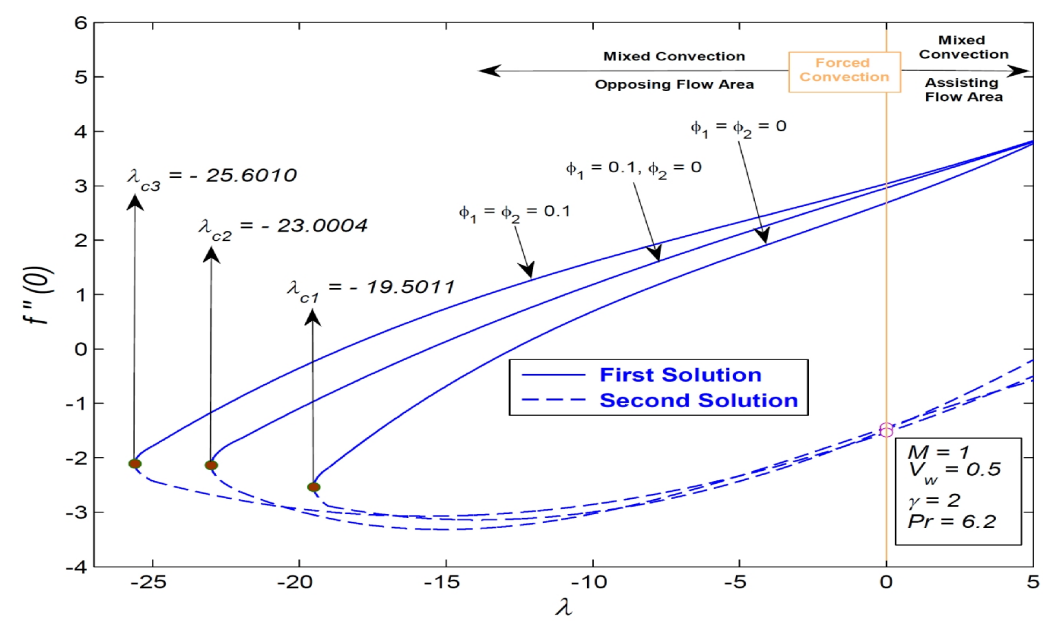

Fig. 2. (Color online) The variation of $f^{\prime \prime}(0)$ as a function of $\lambda$ for different values of $\phi_{1}$ and $\phi_{2}$, when $M=1, V_{w}=0.5, \gamma=2$ and $\operatorname{Pr}=6.2$. 


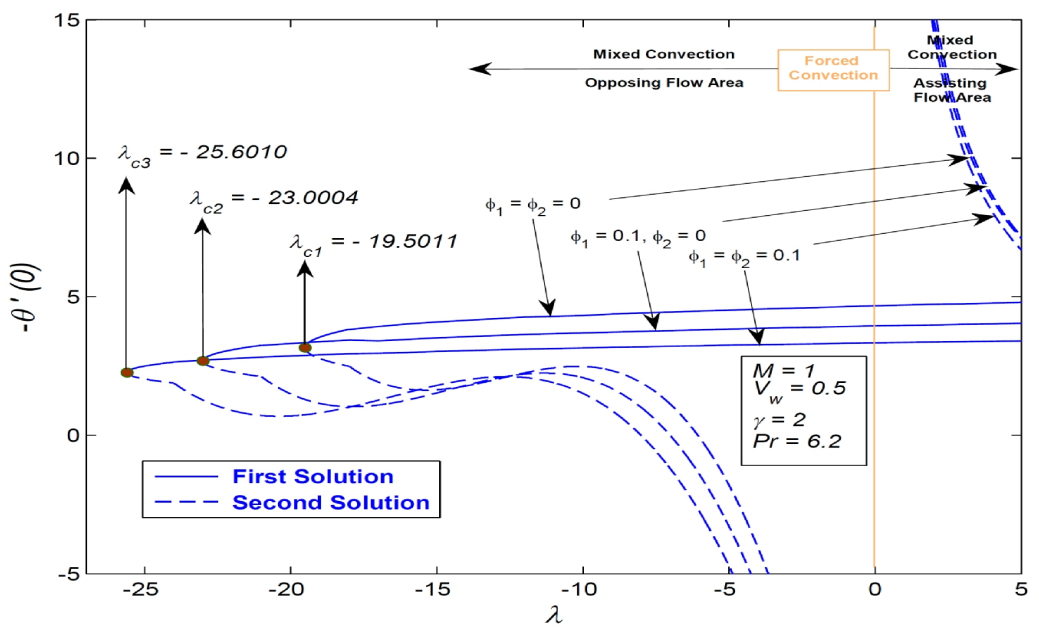

Fig. 3. (Color online) The variation of $-\theta^{\prime}(0)$ as a function of $\lambda$ for different values of $\phi_{1}$ and $\phi_{2}$, when $M=1, V_{w}=0.5, \gamma=2$ and $\operatorname{Pr}=6.2$.

fraction $\mathrm{Cu}$ and $\mathrm{Ag}$. These figures demonstrate that the range of the mixed convection parameter for which the solution exists, increases with nanoparticle volume fraction and it is possible to obtain dual solutions for the similarity Eqs. (6) and (7) also for assisting flow $\lambda>0$, apart of those for opposing flow $\lambda<0$. It is seen that the solution exists up to a critical value of $\lambda$ (that we called $\lambda_{c}$ ), with two solution branches for $\lambda>\lambda_{c}$ (of course except $\lambda=0$ ), a saddle-node bifurcation at $\lambda=\lambda_{c}$ and no solutions for $\lambda<\lambda_{c}$. Therefore, we can conclude from these figures, at $\lambda=0$ (corresponding to forced convection flow), we have only the first solution. Moreover, between these two solutions, we expect that the first solution (upper branch solution) is stable and physically relevant, while the second solution (lower branch solution) is not. It is worth mentioning that, according to authors' knowledge, the present results are the first report of dual solutions for hybrid nanofluid flow.

\subsection{HYDRODYNAMIC AND THERMAL BOUNDARY LAYERS ANALYSIS}

The second solution that physically was unrealizable, so hereafter all of figures graphically have been plotted only for first solutions. Figure 4 illustrates the effect of $M$ on the dimensionless fluid velocity and temperature distributions for two negative (opposing flow) and positive (assisting flow) values of mixed convection parameter $(\lambda)$. The hydrodynamic as well as the thermal boundary layer thicknesses diminish slightly with the increase in the magnetic parameter. According to kind of mixed convection, clearly we can see that there are thinner thermal and hydrodynamic boundary layer thicknesses for assisting flow with respect to opposing flow. The influence of 


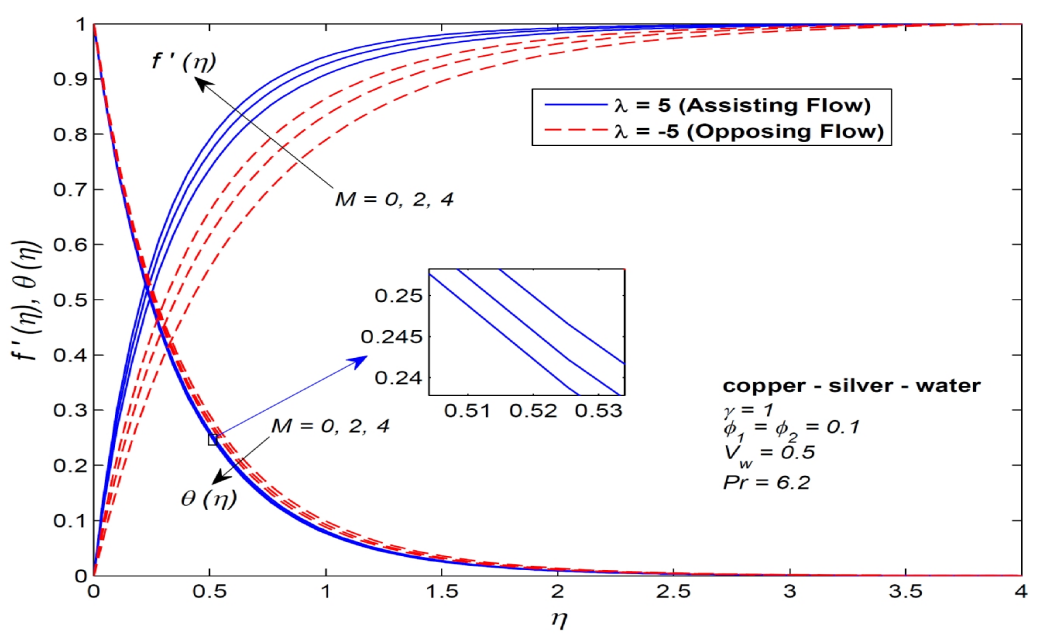

Fig. 4. (Color online) The effect of $M$ on the dimensionless velocity and temperature distributions for assisting and opposing regimes, when $\gamma=1, \phi_{1}=\phi_{2}=0.1, V_{w}=0.5$ and $\operatorname{Pr}=6.2$.

$\gamma$ on the dimensionless fluid velocity and temperature distributions for two negative and positive values of mixed convection parameter is presented in Fig. 5. The results show that there is a point near the cylinder wall where the velocity attains the same

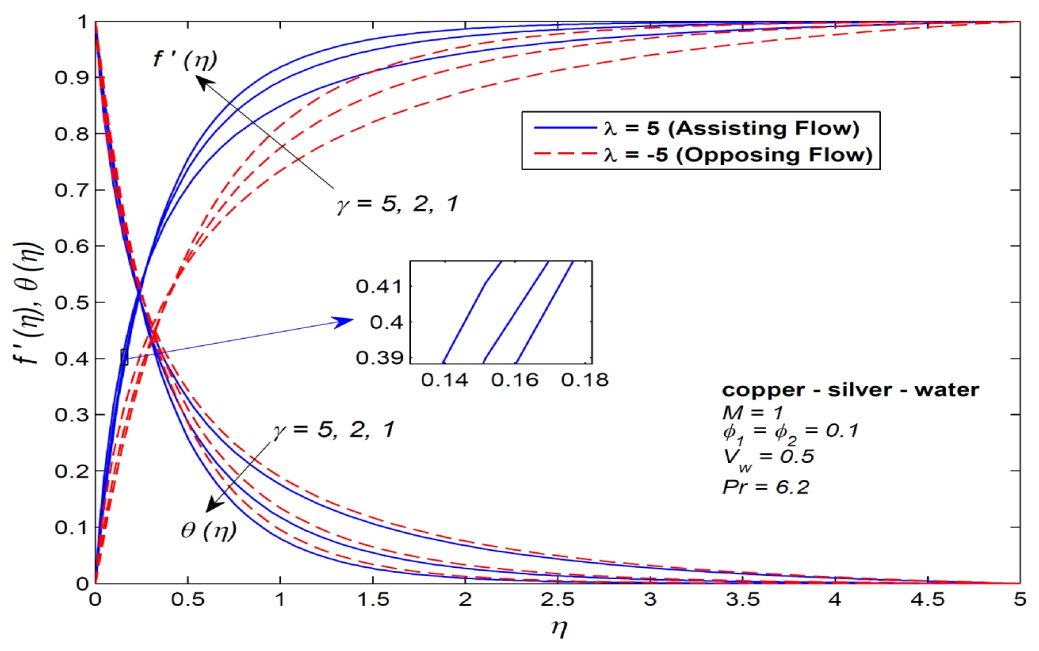

Fig. 5. (Color online) The effect of $\gamma$ on the dimensionless velocity and temperature distributions for assisting and opposing regimes, when $M=1, \phi_{1}=\phi_{2}=0.1, V_{w}=0.5$ and $\operatorname{Pr}=6.2$. 


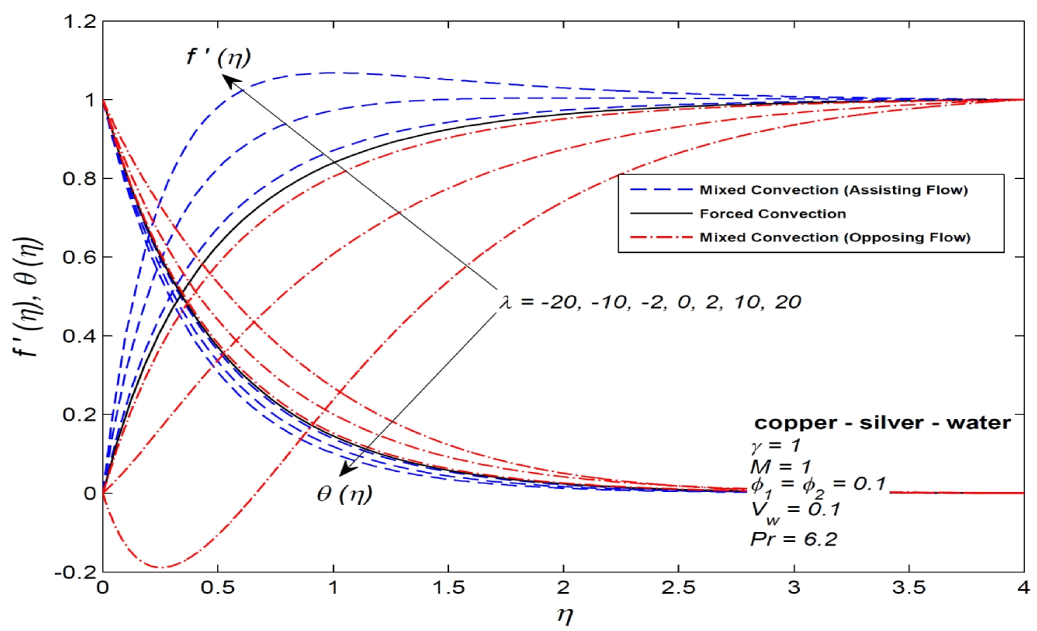

Fig. 6. (Color online) The effect of $\lambda$ on the dimensionless velocity and temperature distributions, when $\gamma=1, M=1, \phi_{1}=\phi_{2}=0.1, V_{w}=0.1$ and $\operatorname{Pr}=6.2$.

value for all values of the curvature parameter. Figure 5 reveals that temperature also decays from a location close to the cylinder wall as we progress into the boundary layer. The changes in the mixed convection parameter consisting three physical cases of assisting flow $(\lambda>0)$, opposing flow $(\lambda<0)$ and forced convective flow $(\lambda=0)$ are presented in Fig. 6. Our results demonstrate that as the positive mixed convection parameter enhances the dimensionless fluid velocity profile increases while the dimensionless temperature distribution decreases slightly. Further, the effect of $M$ and $\lambda$ is comparatively less in dimensionless temperature profiles relative to dimensionless velocity profiles because these parameters does not appear directly in the similarity energy Eq. (7). On the other hand, since the flow field is coupled to the temperature field only by mixed convection parameter, the Grashof number accelerates the fluid so the velocity increases with the increase in $\lambda$. The buoyancy force acts like a favorable pressure gradient and accelerates the fluid, so the velocity increases with the increase in Grashof number and more production occurs. Figure 7 illustrates the effect of the permeability parameter $\left(V_{w}\right)$ on the dimensionless fluid velocity and temperature distributions under determined values of governing parameters. We can see that suction decreases both hydrodynamic and thermal boundary layer thicknesses, while injection is in opposite trend. Besides, it is observed that for suction case decreasing of the thermal boundary layer thickness behavior is stronger relative to the hydrodynamic boundary layer thickness. Further, as we know, in the stagnation point flow, the suction case $\left(V_{w}>0\right)$ has been applied, because the boundary layer thickness is supposed to be practically very thin. 


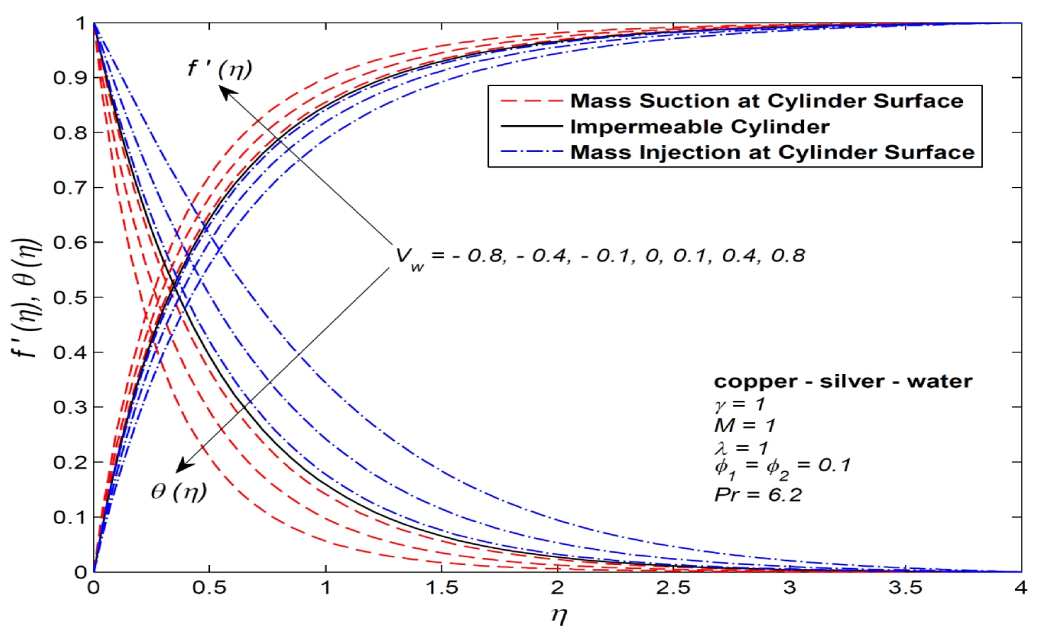

Fig. 7. (Color online) The effect of $V_{w}$ on the dimensionless velocity and temperature distributions, when $\gamma=1, M=1, \lambda=1, \phi_{1}=\phi_{2}=0.1$ and $\operatorname{Pr}=6.2$.

The effect of the second nanoparticle volume fraction (i.e. silver) on the dimensionless velocity profile and temperature distribution is shown in Fig. 8, when $\gamma=M=\lambda=1, \phi_{1}=0.1, V_{w}=0.5$ and $\operatorname{Pr}=6.2$. The hydrodynamic bound-

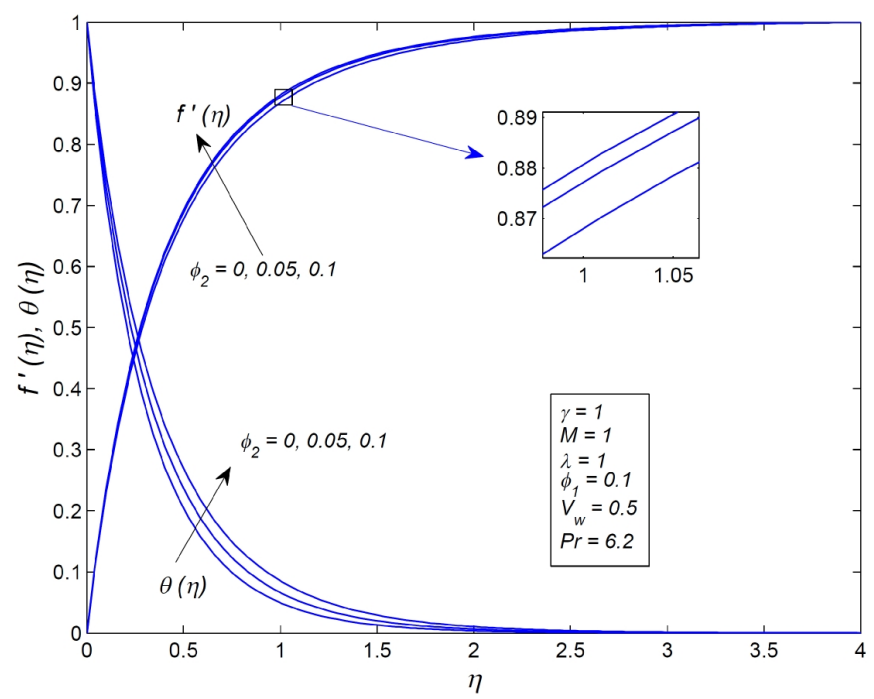

Fig. 8. (Color online) The effect of the second nanoparticle volume fraction $\left(\phi_{\mathrm{Ag}}\right)$ on the dimensionless velocity and temperature distributions, when $\gamma=1, M=1, \lambda=1, \phi_{1}=0.1$, $V_{w}=0.5$ and $\operatorname{Pr}=6.2$. 
ary layer thickness decreases with the second nanoparticle volume fraction while, the dimensionless velocity profile increases. This means the presence of the second nanoparticle leads to further increasing the dimensionless velocity profile in the problem conditions. Moreover, as the thermal conductivity enhances, the thermal boundary layer thickness increases with increasing the second nanoparticle volume fraction. This is in compliance with the primary proposes of employing nanofluids.

\subsection{SKIN FRICTION AND HEAT TRANSFER RATE OF HYBRID NANOFLUID FLOW}

Figures 9-12 present our results for the skin friction coefficient and the local heat transfer rate for a wide range of the curvature parameter $(\gamma)$ and different physical parameters, namely the magnetic parameter $(M)$, the wall permeability parameter $\left(V_{w}\right)$ and the mixed convection parameter $(\lambda)$. All graphs in Figs. 9-12 have been plotted for copper-silver/water hybrid nanofluid when $\phi_{1}=\phi_{2}=0.1$ and $\operatorname{Pr}=6.2$. It can be observed that the value of skin friction coefficient and local heat transfer rate enhances quasi-linearly with increasing the curvature parameter. It is also seen from Figs. 9 and 11, the skin friction coefficient enhances as the magnetic parameter, the wall permeability parameter and the mixed convection parameter (for assisting flow) increase, when $\gamma$ is fixed. However, the skin friction coefficient decreases when values of $|\lambda|$ increase for opposing flow.

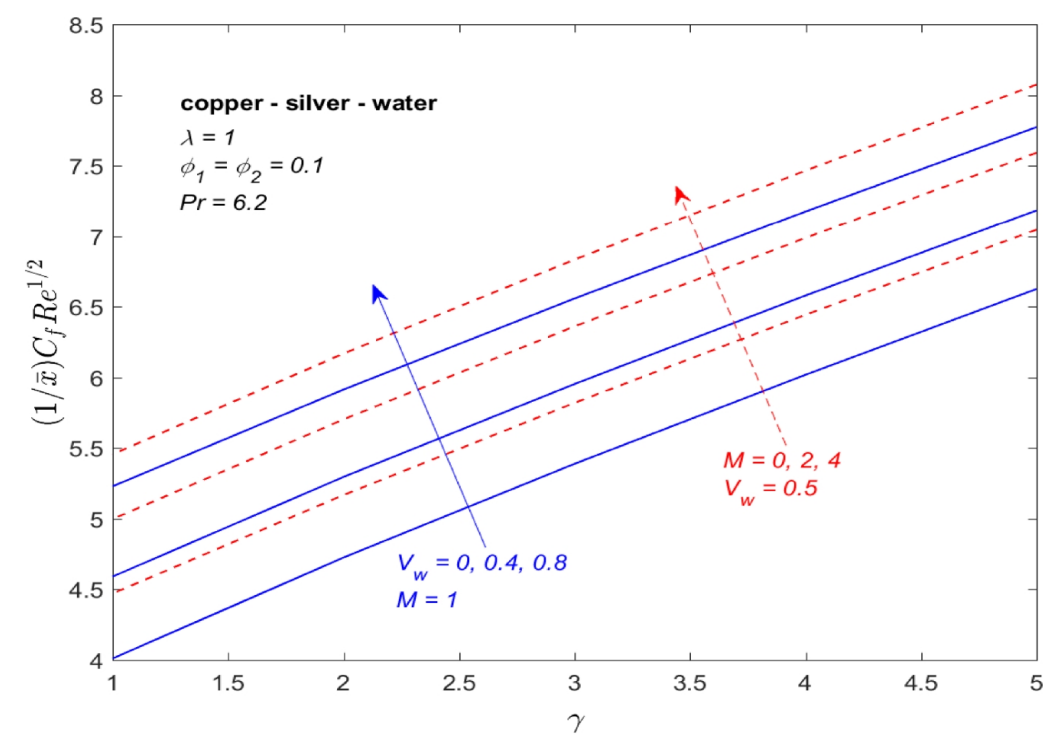

Fig. 9. (Color online) The effect of the magnetic parameter and the wall permeability parameter on the skin friction coefficient, when $\lambda=1, \phi_{1}=\phi_{2}=0.1$ and $\operatorname{Pr}=6.2$. 


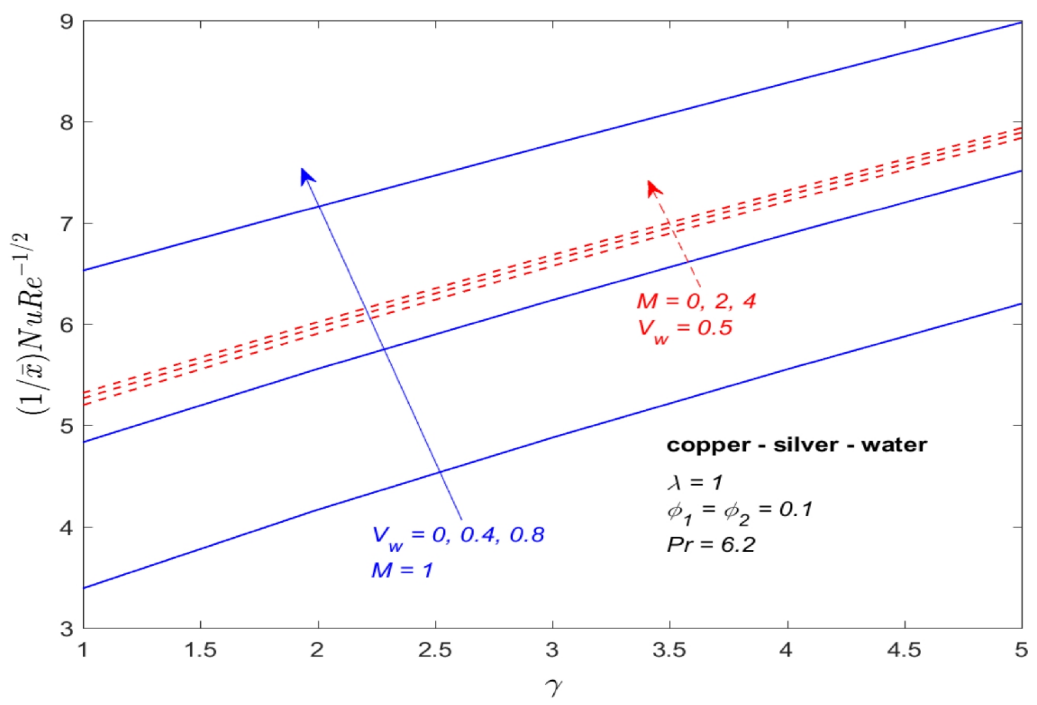

Fig. 10. (Color online) The effect of the magnetic parameter and the wall permeability parameter on the local heat transfer rate, when $\lambda=1, \phi_{1}=\phi_{2}=0.1$ and $\operatorname{Pr}=6.2$.

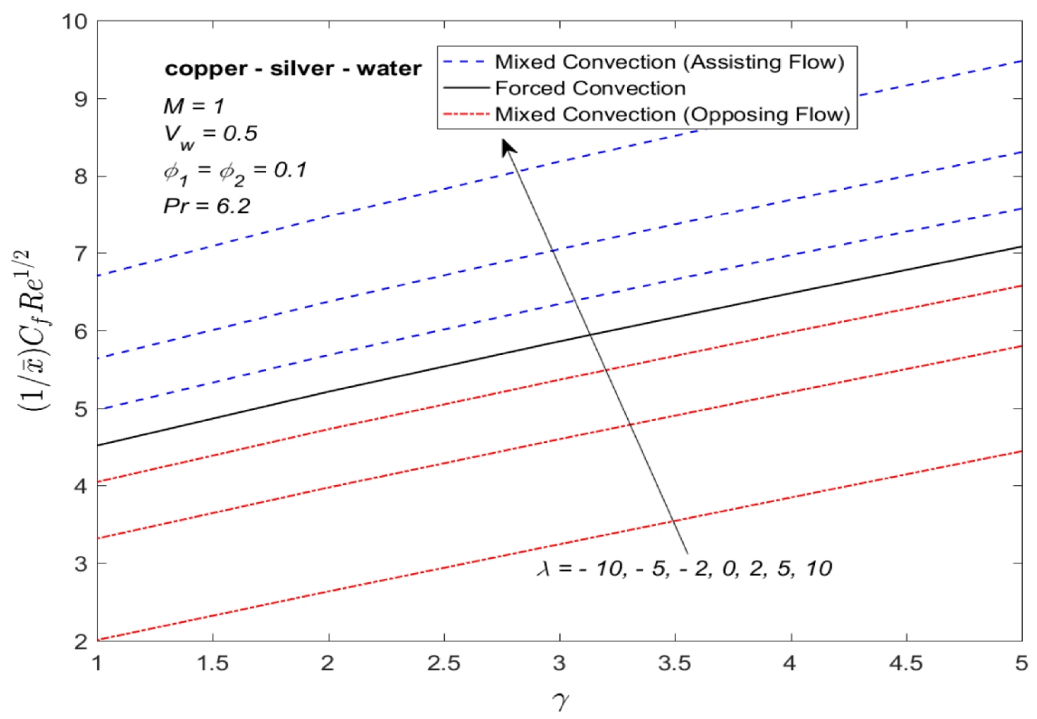

Fig. 11. (Color online) The effect of the mixed convection parameter on the skin friction coefficient when $M=1, V_{w}=0.5, \phi_{1}=\phi_{2}=0.1$ and $\operatorname{Pr}=6.2$. 


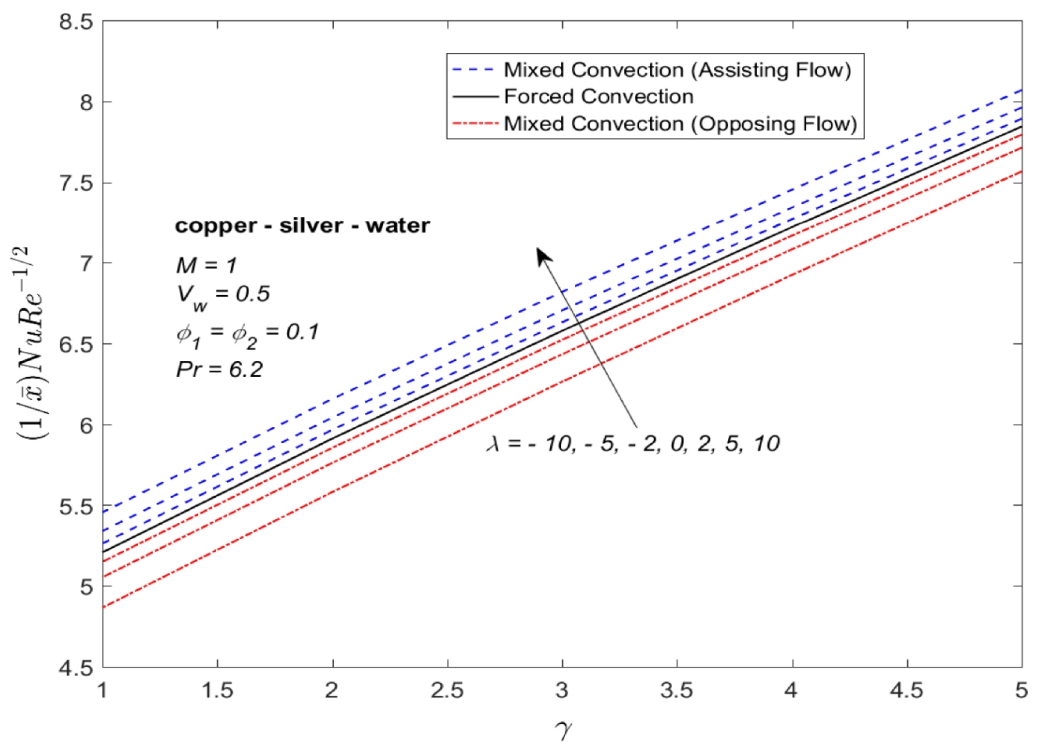

Fig. 12. (Color online) The effect of the mixed convection parameter on the local heat transfer rate when $M=1, V_{w}=0.5, \phi_{1}=\phi_{2}=0.1$ and $\operatorname{Pr}=6.2$.

\subsection{COMPARISON AMONG REGULAR FLUID, MONO-NANOFLUID AND HYBRID NANOFLUID}

In this section, we are going to compare the skin friction coefficient $\left((1 / \bar{x}) R e^{1 / 2} C_{f}\right)$ and the local heat transfer rate $\left((1 / \bar{x}) R e^{-1 / 2} N u\right)$ computed by our new model, for various cases such as the regular fluid (water), nanofluids with single nanoparticles (copper-water and silver-water) and hybrid nanofluid (copper-silver/water) with different values of volume fraction of nanoparticles $\mathrm{Cu}$ and $\mathrm{Ag}$ that have been depicted and indexed in Table 6.

Table 6. The index for Figs. 13 and 14.

\begin{tabular}{llcc}
\hline \hline Types & Description & $\phi_{\mathrm{Ag}}$ & $\phi_{\mathrm{Cu}}$ \\
\hline$R F$ & Regular fluid & 0 & 0 \\
$N F 1$ & Nanofluid (type 1) & 0.05 & 0 \\
$N F 2$ & Nanofluid (type 2) & 0.1 & 0 \\
$N F 3$ & Nanofluid (type 3) & 0 & 0.05 \\
$N F 4$ & Nanofluid (type 4) & 0 & 0.1 \\
$H N F 1$ & Hybrid nanofluid (type 1) & 0.05 & 0.05 \\
$H N F 2$ & Hybrid nanofluid (type 2) & 0.1 & 0.05 \\
$H N F 3$ & Hybrid nanofluid (type 3) & 0.1 & 0.1 \\
\hline
\end{tabular}




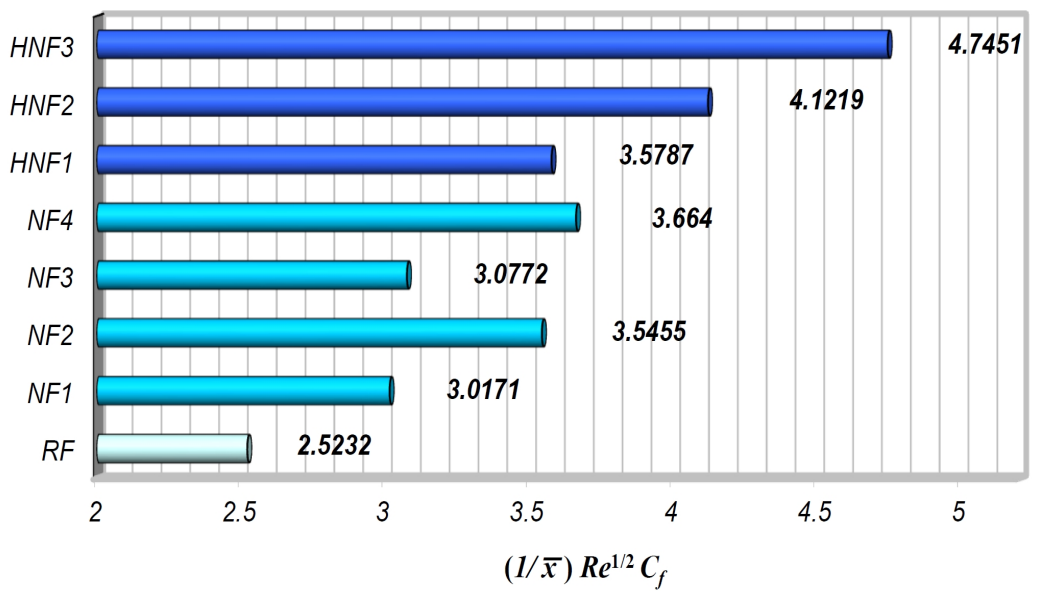

Fig. 13. (Color online) The skin friction coefficient $\left((1 / \bar{x}) R e^{1 / 2} C_{f}\right)$ for various cases of regular fluid, nanofluid and hybrid nanofluid according to Table 6 , when $\gamma=M=\lambda=1$, $V_{w}=0.5$ and $\operatorname{Pr}=6.2$.

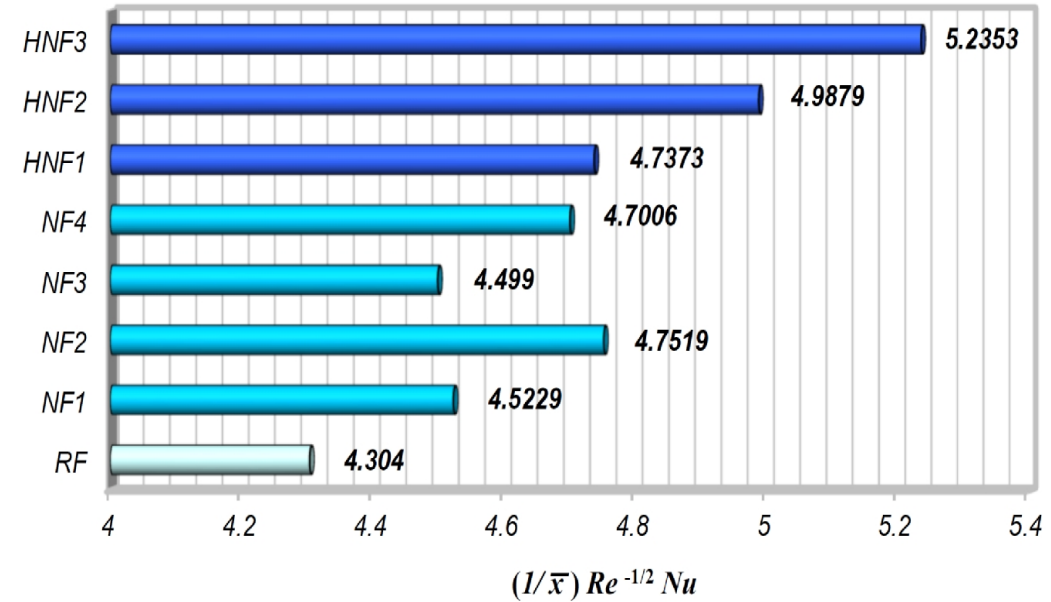

Fig. 14. (Color online) The local heat transfer rate $\left((1 / \bar{x}) R e^{-1 / 2} N u\right)$ for various cases of regular fluid, nanofluid and hybrid nanofluid according to Table 6 , when $\gamma=M=\lambda=1$, $V_{w}=0.5$ and $\operatorname{Pr}=6.2$.

The skin friction coefficient and the local heat transfer rate for various cases, according to Table 6, are plotted in Figs. 13 and 14, respectively, when $\gamma=M=$ $\lambda=1, V_{w}=0.5$ and $\operatorname{Pr}=6.2$. Here, both the skin friction coefficient and the local heat transfer rate enhances with increasing the volume fraction of nanoparticles $\mathrm{Cu}$ and $\mathrm{Ag}$ for all cases. When the volume of nanoparticles enhances the thermal 
conductivity increases then, the thermal boundary layer thickness decreases, so the heat transfer rate rises. In addition, it is obvious that values of the local heat transfer rate and the skin friction coefficient from NF1 and NF3 as well as NF2 and NF4 cases are too near together that is because the thermal conductivity of $\mathrm{Cu}$ and $\mathrm{Ag}$ nanoparticles are too close together (see Table 2). Finally, HNF3 has the largest heat transfer rate $\left((1 / \bar{x}) R e^{-1 / 2} N u=5.2353\right)$ and also the highest skin friction coefficient $\left((1 / \bar{x}) R e^{1 / 2} C_{f}=4.7451\right)$ between all cases. In other words, we can analytically realize that hybrid nanofluids have higher values of local heat transfer rate and skin friction coefficient relative to mono-nanofluids and conventional fluids, respectively.

\section{CONCLUSIONS}

The problem of the steady laminar MHD axisymmetric mixed convective stagnationpoint flow of an incompressible electrically conducting $\mathrm{Cu}-\mathrm{Ag} /$ water hybrid nanofluid along a vertical porous cylinder was studied numerically. This is the first time that an axisymmetric stagnation-point flow by considering a hybrid nanofluid as the advanced working fluid is investigated with taking dual solutions into account. Major Conclusions of the present study can be summarized as follows: 1) dual solutions exist for both assisting and opposing flows of hybrid nanofluid; 2) the first solution (upper branch solution) is stable and physically realizable, while the second solution (lower branch solution) is not; 3) the volume fraction of $\mathrm{Cu}$ and $\mathrm{Ag}$ nanoparticles widen the range of $\lambda$ for which the solution exists; 4) the assisting flow of hybrid nanofluid have lower the hydrodynamic and the thermal boundary layer thicknesses relative to forced convection and opposing flow cases of hybrid nanofluid ones, respectively; 5) by applying the mass suction as well as the magnetic field effects, we can control both hydrodynamic and thermal boundary layer thicknesses; 6) the curvature parameter has a strong additive effect on the skin friction coefficient and the local heat transfer rate; 7) as the optimum selection, when we add both first and second nanoparticles with 5\% volume fraction for each one into the pure water (HNF1 case), the local heat transfer rate enhancement is about $0.77 \%$ higher than dispersing second nanoparticle (lonely) with 10\% volume fraction into it (NF4 case), while the respective skin friction coefficient reduction for the $H N F 1$ case will be $2.38 \%$ relative to the $N F 4$ case; 8) hybrid nanofluids can be suggested to improve the thermophysical properties of regular fluid and mono-nanofluid for heat transfer applications. 


\section{REFERENCES}

[1] M. Muthtamilselvan, S. Sureshrumar (2018) A Tilted Lorentz Force Effect on Porous Media Filled with Nanofluid. Journal of Theoretical and Applied Mechanics 48 50-71.

[2] R. Chand, G.C. RANA, D. YAdAV (2017) Thermal Instability in a Layer of Couple Stress Nanofluid Saturated Porous Medium. Journal of Theoretical and Applied Mechanics 47 69-84.

[3] R.V.M.S.S. Kiran Kumar, S.V.K. VArma (2018) MHD Boundary Layer Flow of Nanofluid Through a Porous Medium Over a Stretching Sheet With Variable Wall Thickness: Using Cattaneo-Christov Heat Flux Model. Journal of Theoretical and Applied Mechanics 48 72-92.

[4] S.K. Das, S.U.S. ChOI, W. Yu, T. PradeEP (2007) "Nanofluids: Science and Technology". Wiley, New Jersey, Canada.

[5] A. Kasaeian, R. Daneshazarian, O. Mahian, L. Kolsi, A.J. Chamkha, S. Wongwises, I. PoP (2017) Nanofluid flow and heat transfer in porous media: A review of the latest developments. International Journal of Heat and Mass Transfer 107 778-791.

[6] R.K. Tiwari, M.K. DAs (2007) Heat Transfer Augmentation in a Two Sided LidDriven Differentially Heated Square Cavity Utilizing Nanofluids. International Journal of Heat and Mass Transfer 50 2002-2018.

[7] J. Buongionno (2006) Convective Transport in Nanofluids. Journal of Heat Transfer 128 240-250.

[8] M.A. Ismael, T. Armaghani, A.J. Chamkha (2016) Conjugate Heat Transfer and Entropy Generation in a Cavity Filled with a Nanofluid-Saturated Porous Media and Heated by a Triangular Solid. Journal of the Taiwan Institute of Chemical Engineers 59 138-151.

[9] A.J. Chamkha, M.A. Ismael (2014) Natural Convection in Differentially Heated Partially Porous Layered Cavities Filled with a Nanofluid. Numerical Heat Transfer Part A 65 1089-1113.

[10] R.S.R. Gorla, A.J. Chamkha (2011) Natural Convective Boundary Layer Flow over a Nonisothermal Vertical Plate Embedded in a Porous Medium Saturated with a Nanofluid. Nanoscale and Microscale Thermophysical Engineering 15 81-94.

[11] A. Zaraki, M. Ghalambaz, A.J. Chamkha, M. Ghalambaz, D. De Rossi (2015) Theoretical Analysis of Natural Convection Boundary Layer Heat and Mass Transfer of Nanofluids: Effects of Size, Shape and Type of Nanoparticles, Type of Base Fluid and Working Temperature. Advanced Powder Technology 26 935-946.

[12] A.A. MineA (2017) Hybrid Nanofluids Based on $\mathrm{Al}_{2} \mathrm{O}_{3}, \mathrm{TiO}_{2}$ and $\mathrm{SiO}_{2}$ : Numerical Evaluation of Different Approaches. International Journal of Heat and Mass Transfer 104 852-860.

[13] S.A.M. Mehryan, F.M. Kashrooli, M. Ghalambaz, A.J. Chamkha (2017) Free Convection of Hybrid $\mathrm{Al}_{2} \mathrm{O}_{3}-\mathrm{Cu}$ Water Nanofluid in a Differentially Heated Porous Cavity. Advanced Powder Technology 28 2295-2305. 
[14] A.J. Chamkha, I.V. Miroshnichenko, M.A. Sheremet (2017) Numerical Analysis of Unsteady Conjugate Natural Convection of Hybrid Water-Based Nanofluid in a Semicircular Cavity. Journal of Thermal Science and Engineering Applications 9 041004-9.

[15] M. Ghalambaz, M.A. Sheremet, S.A.M. Mehryan, F.M. Kashkooli, I. Pop (2019) Local Thermal Non-Equilibrium Analysis of Conjugate Free Convection within a Porous Enclosure Occupied with Ag-MgO Hybrid Nanofluid. Journal of Thermal Analysis and Calorimetry 135 1381-1398.

[16] M. Izadia, R. Mohebbi, D. Karimi, M.A. Sheremet (2018) Numerical Simulation of Natural Convection Heat Transfer Inside a + Shaped Cavity Filled by a MWCNT-Fe ${ }_{3} \mathrm{O}_{4} /$ Water Hybrid Nanofluids Using LBM. Chemical Engineering \& Processing: Process Intensification 125 56-66.

[17] De-Yi Shang, LiAng-CAi Zhong (2016) "Heat Transfer of Laminar Mixed Convection of Liquid", SpringerNature, Switzerland.

[18] S. Dinarvand, A. AbBassi, R. Hosseini, I. Pop (2015) Homotopy Analysis Method for Mixed Convective Boundary Layer Flow of a Nanofluid over a Vertical Circular Cylinder. Thermal Science 19 549-561.

[19] N. Ramachandran, T.S. Chen, B.F. Armaly (1988) Mixed Convection in Stagnation Flows Adjacent to a Vertical Surface. ASME Journal of Heat Transfer 110373 377.

[20] A. IsHAK, R. NAZAR, N. BACHOK, I. POP (2010) MHD Mixed Convection Flow near the Stagnation-Point on a Vertical Permeable Surface. Physica A 389 40-46.

[21] H. Tamim, S. Dinarvand, R. Hosseini, S. Khalili, I. Pop (2014) Unsteady Mixed Convection Flow of a Nanofluid near Orthogonal Stagnation-Point on a Vertical Permeable Surface. Journal of Process Mechanical Engineering 228 226-237.

[22] E. Abu-NadA, A.J. Chamkha (2014) Mixed Convection Flow of a Nanofluid in a Lid-Driven Cavity with a Wavy Wall. International Communications in Heat and Mass Transfer 57 36-47.

[23] F. Selimefendigil, H.F. Öztop, A.J. Chamkha (2016) MHD Mixed Convection and Entropy Generation of Nanofluid Filled Lid Driven Cavity under the Influence of Inclined Magnetic Fields Imposed to Its Upper and Lower Diagonal Triangular Domains. Journal of Magnetism and Magnetic Materials 406 266-281.

[24] Ch. RamReddy, P.V.S.N. Murthy, A.J. Chamkha, A.M. Rashad (2013) Soret Effect on Mixed Convection Flow in a Nanofluid under Convective Boundary Condition. International Journal of Heat and Mass Transfer 64 384-392.

[25] S. Dinarvand, R. Hosseini, I. Pop (2017) Axisymmetric Mixed Convective Stagnation-Point Flow of a Nanofluid over a Vertical Permeable Cylinder by TiwariDas Nanofluid Model. Powder Technology 311 147-156.

[26] A. RIDHA (1996) Aiding Flows Non-Unique Similarity Solutions of Mixed-Convection Boundary-Layer Equations. Zeitschrift für Angewandte Mathematik und Physik 47341 352 . 
[27] N. SAndeEP, I.L. Animas Aun (2017) Theoretical Exploration of Exponential Heat Source and Thermal Stratification Effects on the Motion of 3-Dimentional Flow of Casson Fluid over a Low Heat Energy Surface at Initial Unsteady Stage. Journal of Theoretical and Applied Mechanics 47 61-82.

[28] M. Nademi Rostami, S. Dinarvand, I. Pop (2018) Dual Solutions for Mixed Convective Stagnation-Point Flow of an aqueous Silica-Alumina Hybrid Nanofluid. Chinese Journal of Physics 56 2465-2478.

[29] M. Yousefi, S. Dinarvand, M. Eftekhari Yazdi, I. Pop (2018) StagnationPoint Flow of an Aqueous Titania-Copper Hybrid Nanofluid toward a Wavy Cylinder. International Journal of Numerical Methods for Heat \& Fluid Flow 28 1716-1735.

[30] S. NADEEm, N. ABBAS, A.U. Khan (2018) Characteristics of Three Dimensional Stagnation Point Flow of Hybrid Nanofluid Past a Circular Cylinder. Results in Physics 8 829-835.

[31] T. Hayat, S. NadeEm (2017) Heat Transfer Enhancement with Ag-CuO/Water Hybrid Nanofluid, Results in Physics 7 2317-2324.

[32] S. DinARVAND, I. Pop (2017) Free-Convective Flow of Copper/Water Nanofluid about a Rotating Down-Pointing Cone Using Tiwari-Das Nanofluid Scheme. Advanced Powder Technology 28 900-909.

[33] D. Mott, J. Galkowski, L. Wang, J. Luo, Chuan-Jian Zhong (2007) Synthesis of Size-Controlled and Shaped Copper Nanoparticles. Langmuir 23 5740-5745.

[34] B. Khodashenas, H.R. Ghorbani (2015) Synthesis of Silver Nanoparticles with Different Shapes. Arabian Journal of Chemistry DOI: https://doi.org/10.1016/j.arabjc.2014.12.014.

[35] M. Dubiel, S. Brunsch, L. Tröger (2001) Temperature Dependence of Thermal Expansion Coefficient of Silver Nanoparticles and of Bulk Material Determined by EXAFS. Journal of Synchrotron Radiation 8 539-541.

[36] T. Mahmood, J.H. Merkin (1988) Similarity Solutions in Axisymmetric Mixed Convection Boundary-Layer Flow. Journal of Engineering Mathematics 22 73-92.

[37] L.F. Shampine, I. Gladwell, S. Thompson (2003) "Solving ODEs with MATLAB". Cambridge University Press, Cambridge, England.

[38] T. Grosan, I. Pop (2011) Axisymmetric Mixed Convection Boundary Layer Flow Past a Vertical Cylinder in a Nanofluid. International Journal of Heat and Mass Transfer 54 3139-3145. 